\title{
AN EPISTEMIC NETWORK ANALYSIS OF PATIENT DECISION-MAKING REGARDING CHOICE OF THERAPY
}

Zörgő S, Peters GJY, Csajbók-Veres K, Geröly A, Jeney A, Ruis AR

\section{Post peer review preprint}

This manuscript has been accepted to the journal Quality \& Quantity. Please check back here for correct citation in 2022. 


\title{
AN EPISTEMIC NETWORK ANALYSIS OF PATIENT DECISION-MAKING REGARDING CHOICE OF THERAPY
}

\author{
${ }^{1}$ Zörgő S四 [0000-0002-6916-2097], ${ }^{2}$ Peters GJY [0000-0002-0336-9589], , ${ }^{3}$ Csajbók-Veres K, ${ }^{3}$ Geröly A, ${ }^{4}$ Jeney A [0000-0002-6037-9505], ${ }^{5}$ Ruis AR \\ [0000-0003-1382-4677] \\ 1 Institute of Behavioral Sciences, Semmelweis University, Budapest, Hungary \\ zorgoszilvia@gmail.com \\ 2 Faculty of Psychology \& Education Science, Open University, Heerlen, Netherlands \\ 3 Center for Intercultural Psychology and Education, Eötvös Loránd University, Budapest, Hungary \\ 4 Department of Cultural Anthropology, Eötvös Loránd University, Budapest, Hungary \\ 5 Wisconsin Center for Education Research, University of Wisconsin-Madison, USA
}

\begin{abstract}
Background: Patient decision-making concerning therapy choice has been thoroughly investigated in the Push/Pull framework: factors pushing the patient away from biomedicine and those pulling them towards Complementary and Alternative Medicine (CAM). Others have examined lay etiology as a potential factor in CAM use.

Methods: We conducted semi-structured interviews with patients employing only biomedicine and those using CAM. The coded and segmented data was quantified and modelled using epistemic network analysis (ENA) to explore what effects push/pull factors and etiology had on the decision-making processes.

Results: There was a marked difference between our two subsamples concerning push factors: although both groups exhibited similar scaled relative code frequencies, the CAM network models were more interconnected, indicating that CAM users expressed dissatisfaction with a wider array of phenomena. Among pull factors, a preference for natural therapies accounted for differences between groups but did not retain a strong connection to rejecting conventional treatments. Etiology, particularly adherence to vitalism, was also a critical factor in both choice of therapy and rejection of biomedical treatments.

Conclusions: Push factors had a crucial influence on decision-making, not as individual entities, but as a constellation of experienced phenomena. Belief in vitalism affects the patient's explanatory model of illness, changing the interpretation of other etiological factors and illness itself. Scrutinizing individual push/pull factors or etiology does not explain therapeutic choices; it is from their interplay that decisions arise. Our unified, qualitative-and-quantitative methodological approach offers novel insight into decision-making by displaying connections among codes within patient narratives.
\end{abstract}

Keywords: Complementary and Alternative Medicine, Decision-making, Push and Pull factors, Lay etiology, Qualitative, Epistemic Network Analysis 


\section{BACKGROUND}

Complementary and Alternative Medicine (CAM) is an umbrella term for various non-conventional medical modalities: "A broad domain of healing resources that encompasses all health systems, modalities, and practices and their accompanying theories and beliefs, other than those intrinsic to the politically dominant health systems of a particular society or culture in a given historical period" (Wieland et al., 2011). CAM modalities, like any other healthcare practice, are not merely a product or service, but are embedded in a larger cultural system with specific values and practices. These modalities can be used instead of biomedicine (alternative use) or in conjunction with conventional medicine (complementary use). When biomedicine is the dominant medical modality in a country's healthcare system, CAM is most commonly employed to treat chronic diseases, especially cancer (up to 93\%) (Keene et al., 2019) and musculoskeletal diseases (Eardley et al., 2012).

Many studies have explored sociodemographic characteristics in connection with CAM use in Western countries, concluding that the modal CAM user is a middle-aged, well-educated, Caucasian female (Arthur et al., 2012; Harris, 2012; Hunt et al., 2010). Reasons behind CAM use have been investigated via patient motivations. The market niche hypothesis, for example, claims that CAM appeals to patients in areas where biomedicine is perceived to be lacking, such as patient-centered care and the attribution of meaning to suffering (Cartwright \& Torr, 2005; Knoll, 2004; White \& Verhoef, 2006). In another approach, Boon et al. group pertinent considerations into fixed decision factors that the patient cannot change (e.g., demographic characteristics, comorbid conditions, disease status) and flexible decision factors (e.g., patient perceptions of conventional and CAM treatments, psychological needs) (Boon et al., 2003). Many CAM researchers subscribe to the push/pull dichotomy, examining patient decision-making in terms of push factors repelling patients from biomedicine and pull factors drawing patients toward CAM (Furnham \& Vincent, 2000). Common push factors include perceived failures of biomedicine (patient cannot be diagnosed or cured with conventional treatments), negative experiences with conventional cures (ineffectiveness or severe side-effects), and dissatisfaction with biomedicine in general or with a specific practitioner. The most prominent pull factor is philosophical congruence (Vincent \& Furnham, 1996), signifying that a patient employs CAM because they believe they have identified their own cultural values in the sociocultural environment of a CAM modality. As CAM practices are usually embedded in a distinctive sociocultural system, these values can take on many dispositions: cognitive or behavioral tendencies (Geertz, 1973). A prominent disposition among CAM users is a preference for the "natural", generally defined as "clean", "healthy", "not man-made" products or procedures. The natural may also manifest in dietary choices, such as consuming "organic" or "unprocessed" food and avoiding "toxins". (Bishop et al., 2007; Stratton \& McGivern-Snofsky, 2008; White \& Verhoef, 
Additionally, lay theories of illness causation, especially psychosocial etiologies, have been found to predict CAM use (Bishop et al., 2007; Maskarinec et al., 2001). In these etiologies, a (largely) unidirectional causality between soul/mind and body enables psychosocial phenomena to cause somatic illness (Arnault, 2009; Siahpush, 1999; Zörgő \& Olivas Hernández, 2018). This belief is intimately tied to psychologization, dominant in the broader healthcare market of Western countries, where healing is conceptualized as the transformation of Self through illness or "personal growth" (Frank, 1993; Reddy, 2002; Thompson, 2003; Thorne et al., 2002; White \& Verhoef, 2006). Another type of etiology, vitalism, has also been found to correlate with CAM. (Bishop et al., 2007; Goldstein, 2002; Zörgő \& Olivas Hernández, 2018) Belief in concepts of vital (or spiritual) energy may often constitute part of the "spirituality" dimension in quantitative surveys (Thomson et al., 2014), but it is not necessarily identical. According to vitalism, a "universal energy" courses through or gives rise to all living things, which connects or unifies "consciousness/es". In this worldview, social relationships and life events (including illness) occur in order to "teach" the individual or for other teleological reasons. Vitalism may be associated with beliefs accredited to Eastern philosophy, such as karma and reincarnation.

Many scholars have argued that the effects of dispositions or etiology eclipse patient dissatisfaction in motivations underlying CAM use (Arthur et al., 2012; Astin, 1998; Siahpush, 1999). Yet, these assumptions are based on research designs that usually do not differentiate among patient groups and rely on surveys to measure either lay etiology or push/pull factors regarding CAM use. The interplay among these three areas in patient journeys and explanatory models is most aptly investigated with qualitative methods, yet the three domains have not been modelled in a way that captures the relation of such constructs to rejecting conventional treatment. As we were interested in both general and illness-specific tendencies, our inquiry necessitated an analytical method that depicts the complex interplay of all these factors.

Epistemic Network Analysis (ENA) is a tool for constructing dynamic network models that measure and visualize the structure of connections among elements of complex thinking in discourse data (D. Shaffer, 2017). ENA has been used in healthcare-related research by, for example, Sullivan et al. to identify targets for educational interventions in trauma team communication (Sullivan et al., 2018), Ruis et al. to evaluate intraoperative performance among general surgery residents (A. R. Ruis et al., 2018), Wooldridge et al. to assess communication in primary care teams during patient handoffs (Wooldridge \& Haefli, 2019), and Shum et al. to model teamwork, critical thinking, and clinical skills during nursing team simulations (Buckingham Shum, Simon et al., 2019).

Our objective was to gain an understanding of phenomenological factors in patient decision-making through investigating how therapy choice and rejecting conventional cures interact with a) lay theory of illness causation (etiology), b) push factors (negative experiences), and c) pull factors (dispositions) in narratives from users of biomedicine only (B) and users of complementary and alternative medicine (CAM) among three illness groups (diabetes, musculoskeletal diseases, and digestive illnesses). 


\section{METHODS}

\section{Terminology}

Users of biomedicine were defined as patients solely employing conventional biomedical treatments; even if the individual had tried a CAM modality once earlier but subsequently did not employ CAM, they were included in the biomedicine subsample. Participants in the CAM group were composed of individuals using one or more CAM modalities along with or instead of biomedicine. CAM was defined as healing modalities that are not typically accepted by conventional medicine practitioners and are not covered by social security; CAM use was defined as employing a non-conventional modality through a CAM practitioner or a CAM product that was explicitly stated as non-conventional or alternative by the participant.

\section{Data collection}

In order to explore patient decision-making, we conducted semi-structured interviews. Non-proportional quota sampling was employed, stratifying on therapy choice (B and CAM), primary diagnosis (D1, D2, D3, D4), and sex (male and female). Inclusion criteria were the following: 18 years of age or above; having received a diagnosis of D1-D4; and resident of Budapest, Hungary. Four nosological groups were included: D1 - Diabetes (I, II, insulin resistance), D2 - Musculoskeletal diseases, D3 - Digestive illnesses, and D4 - Nervous system diseases. Inclusion based on primary diagnosis was defined by the patient having received a diagnosis from a conventional doctor based on biomedical test results. Data collection began in February 2019; four researchers conducted the interviews who had all been trained in qualitative methods and in employing our specific data collection tool. Interviews lasted for 60 minutes on average, ranging from 40 to 120 minutes; they were audio-recorded, transcribed verbatim, and anonymized. Table 1 shows the main topics of the semi-structured interview and their subtopics. For each participant, we recorded the following attributes (properties/characteristics of patients): sex, age, level of education, diagnosis type (D1-D4), specific illness, comorbidities, illness onset, time of diagnosis, and therapy choice (treatment type concerning primary diagnosis). Informed consent was obtained from all participants. The ethics permit was issued by Semmelweis University Regional and Institutional Committee of Science and Research Ethics (SE RKEB: 226/2018).

Table 1. Areas of the semi-structured interview and number of related codes.

\begin{tabular}{l|l|c} 
PARENT CODE & \multicolumn{1}{|c}{ CONTENT } & QUESTION LOAD \\
\hline EPISTEMOLOGY & $\begin{array}{l}\text { Information } \\
\text { sources of health-related information, appraisal of information }\end{array}$ & 6 questions + probes \\
\hline ONTOLOGY & $\begin{array}{l}\text { Explanatory Model } \\
\text { concepts of illness and health, etiology }\end{array}$ & 4 questions + probes \\
\hline BEHAVIOR & $\begin{array}{l}\text { Patient journey } \\
\text { choices of therapy, evaluation of therapeutic efficacy }\end{array}$ & 5 questions + probes
\end{tabular}




\section{Discourse coding and segmentation}

We employed deductive coding; codes were adopted from a previous, fully qualitative study on the same topic (Zörgő et al., 2018). Our coding system contained three levels of hierarchy, with a total of 52 low-level codes, 19 of which were used in the present study. Coding was performed by three researchers; each researcher "specialized" in one of the three high-level codes. Coding was preceded by a training period during which subsets of the raw data were coded independently and then triangulated; code interpretations were refined as needed. Subsequently, we created the final version of the codebook, and coded the narrative corpus deductively with those codes. Even though patients were included into the study based on a primary diagnosis, they rarely suffered from a single disease and thus often discussed comorbidities as well. Codes were applied to narratives regardless of which disease the patient was referring to. During the process of coding, several decisions were made about unique manifestations of codes and narrative segments where assigning a code was more ambiguous compared to other instances. These decisions and their justifications were logged in a separate document, which, along with a detailed codebook, a rationale for our selection of codes for this study, our data collection instruments, and other materials can be found in our repository (available at: https://osf.io/7sm5n/). Codes elaborated in our present study and their description can be found in Table 2.

Table 2. Codes analyzed in our current study $(n=19)$ and their description.

\begin{tabular}{|c|c|c|c|}
\hline PARENT & CHILD & GRANDCHILD & DESCRIPTION \\
\hline \multirow{5}{*}{$\begin{array}{l}\text { Etiology } \\
(\mathrm{N}=5)\end{array}$} & \multirow{5}{*}{ Illness causation } & Psychosocial & Emotion, stress, trauma, nerves (no mention of energy) \\
\hline & & Vitalistic & Energy/qi/prana, flow, block, law of attraction, nothing is by chance \\
\hline & & Ecological & Environmental toxins, chemicals, "electro-smog" \\
\hline & & Nutritional & Quality or type of food, additives \\
\hline & & Genetic & Inherited illness or susceptibility, genes, runs in the family \\
\hline \multirow{6}{*}{$\begin{array}{l}\text { PUSH FACTORS } \\
(\mathrm{N}=6)\end{array}$} & \multirow{3}{*}{ Lack } & No diagnosis & Patient received no diagnosis; doctors don't know what's wrong \\
\hline & & No choice & Had no other choice but to employ the conventional treatment \\
\hline & & No cure & Biomedical cure doesn't exist or is unavailable \\
\hline & \multirow{3}{*}{ Negative experiences } & Dissatisfaction & Dissatisfaction with a physician or conventional medicine \\
\hline & & Cure ineffective & Conventional treatment was not effective \\
\hline & & Side-effects & Biomedical treatment or procedure had side-effects \\
\hline \multirow{6}{*}{$\begin{array}{l}\text { PULL FACTORS } \\
(\mathrm{N}=6)\end{array}$} & \multirow{3}{*}{ Judging information } & Intuition & "Inner compass”, "gut feeling”, "just know”, "experience”, etc. \\
\hline & & Science & "Evidence", "scientific proof", statistics, etc. \\
\hline & & Natural & "Natural" (how individual defined it) and objects it refers to \\
\hline & \multirow{3}{*}{ Appraisal of therapeutic efficacy } & Measured & Appraisal based on biomedical test result (blood test, CT, MRI, etc.) \\
\hline & & Interoceptive & Appraisal based on a bodily sensation \\
\hline & & Temporality & Appraisal based on speed (slow or fast) \\
\hline \multirow{2}{*}{$\begin{array}{l}\text { DECISION } \\
(N=2)\end{array}$} & \multirow{2}{*}{$\begin{array}{l}\text { Rejection of conventional } \\
\text { treatment }\end{array}$} & Reject Prior & Biomedical intervention rejected a priori before it was tried (i.e.: forgo) \\
\hline & & Reject Post & Biomedical intervention rejected a posteriori after it was tried (i.e.: discontinue) \\
\hline
\end{tabular}

In order to attain meaningful code co-occurrences, sources of data (i.e., interview transcripts) were segmented. Sentences constituted the smallest meaningful unit of segmentation in our project (i.e., utterances). Each sentence was separated by a line break 
and received a unique utterance identifier (UID); coding occurred on this level of segmentation. Mid-level segmentation (i.e., recent temporal context) was applied in the case of push factors, but not concerning pull factors and etiology. The latter two code clusters attempted to capture the patient's value system and explanatory model of illness, indicating that more distal co-occurrences (such as a patient judging the efficacy of a treatment by "giving it time" and "tracking how symptoms change") are valid connections, no matter where they occur in the interview transcript. Push factor codes referred to discrete events along the patient journey, such as experiencing side-effects and expressing dissatisfaction about that, thus, in this case, tighter psychological proximity was warranted. For this reason, two researchers independently segmented all transcripts delimiting events in the patient journey. Differences in segmentation were resolved through triangulation.

Coding and segmentation were performed in compliance with the Reproducible Open Coding Kit (ROCK) standard that facilitates transparent qualitative research. We employed iROCK (a browser-based graphical interface) to code our sources manually, then, based on the UIDs, the coded data was aggregated with the rock R package. In this process, discourse coding was converted to binary representation ( 1 if a code occurs in a particular utterance, 0 if it does not). Thus, each line in our final dataset contained an utterance, the coded data in binary form, and patient attributes.

\section{Epistemic Network Analysis (ENA)}

ENA methodology is described in detail elsewhere (Bowman et al., 2021; D. Shaffer, 2017; D. W. Shaffer et al., 2016), but in

brief, ENA calculates the co-occurrence of each unique pair of codes within a given segment of discourse and aggregates this information for each patient (i.e. unit of analysis) in a cumulative adjacency matrix, which is represented as a vector in a highdimensional space. Because the length of the vectors is potentially affected by the length of the narratives, each unit vector is divided by its own length in the process of spherical normalization; the resulting normalized vector thus quantifies the relative frequencies of co-occurrence among codes independent of discourse length. This process also transforms connection strengths within the networks to numbers between zero and one. ENA then projects the networks as points into a low-dimensional space. In this study, regarding all models, we projected networks into a two-dimensional space in which the first $(\mathrm{x})$ dimension was constructed using a means rotation $(\mathrm{MR})$, which maximizes the distance between two pre-defined groups (in this case, B and CAM), and the second (y) dimension was constructed using singular value decomposition (SVD), which maximizes the remaining variance explained. Network nodes are then placed in fixed locations in the low-dimensional space using a least-squares method that minimizes the distances between the locations of the networks in the projected space and the centroids of their corresponding weighted network graphs. The result is two, coordinated representations of the data: 1) network graphs, where the nodes in the model correspond to the codes in the discourse 
and the edges represent the relative strength of connection among codes, and 2) ENA scores, or the position of each network in the low-dimensional space. The structure of each network can thus be used to explain the location of the unit in the reduced space. ENA uses the following two parameters to operationalize relational context for each unit of analysis: conversation and stanza window. Conversations are groupings of utterances that can be connected in a model; in our case, codes could co-occur within mid-level segmentation (in the case of push factor codes) or within an entire interview, or source (in the case of pull factor and etiology codes). Code co-occurrences were aggregated within each diagnosis group for both biomedicine and CAM users separately. For all models, code co-occurrences were computed using a weighted whole conversation stanza window (mode of accumulation): codes occurring anywhere within the same conversation were considered to be connected, and the weights of the connections were determined by the number of times each code occurred within the conversation. Network model parameterization is described in Table 3.

Table 3. Parameters of the three network configurations generated with Epistemic Network Analysis (ENA).

\begin{tabular}{|c|c|c|c|}
\hline & $\begin{array}{l}\text { Etiology and Rejection of } \\
\text { Conventional Treatment }\end{array}$ & $\begin{array}{c}\text { Push factors and Rejection of } \\
\text { Conventional Treatment }\end{array}$ & $\begin{array}{c}\text { Pull factors and Rejection of } \\
\text { Conventional Treatment }\end{array}$ \\
\hline UNIT & Group > Diagnosis group > Source & Group > Diagnosis group $>$ Source & Group > Diagnosis group > Source \\
\hline CONVERSATION & Group > Diagnosis group > Source & $\begin{array}{c}\text { Group > Diagnosis group > Source > Mid- } \\
\text { level segmentation }\end{array}$ & Group > Diagnosis group > Source \\
\hline STANZA WINDOW & Whole conversation (weighted) & Whole conversation (weighted) & Whole conversation (weighted) \\
\hline CODES & $\begin{array}{c}\text { Psychosocial, Vitalistic, Genetic, } \\
\text { Nutritional, Ecological, Reject prior, Reject } \\
\text { post }\end{array}$ & $\begin{array}{l}\text { No diagnosis, No Choice, No cure, } \\
\text { Dissatisfaction, Cure ineffective, Side- } \\
\text { effects, Reject prior, Reject post }\end{array}$ & $\begin{array}{c}\text { Intuition, Science, Natural, Measured, } \\
\text { Interoceptive, Temporal, Reject prior, } \\
\text { Reject post }\end{array}$ \\
\hline Projection & $\begin{array}{c}\text { MR1: } 13.4 \% \text {; SVD2: } 18.3 \% \text { (together } \\
\text { account for } 31.7 \% \text { of the total variance in } \\
\text { the data) }\end{array}$ & $\begin{array}{c}\text { MR1: } 12.3 \% \text {; SVD2: } 15.6 \% \text { (together } \\
\text { account for } 27.9 \% \text { of the total variance in } \\
\text { the data) }\end{array}$ & $\begin{array}{c}\text { MR1: } 23.9 \% \text {; SVD2: } 19.8 \% \text { (together } \\
\text { account for } 43.7 \% \text { of the total variance in } \\
\text { the data) }\end{array}$ \\
\hline
\end{tabular}

\section{RESULTS}

\section{Sample characteristics}

Our sample consisted of 26 patients ( 8 male, 18 female); most individuals were between the ages of 20 and 39 . Two subsamples were created based on therapy choice: users of biomedicine only $(n=13)$ and users of CAM ( $n=13)$. Higher education (bachelor's degree, master's degree, or doctorate) was slightly more frequently reported in the biomedicine group ( $\mathrm{n}=11$ ) than the CAM group $(n=9)$. The distribution of primary diagnoses with which patients were included into the study is displayed in Table $4 ; 20$ patients were diagnosed in adulthood, while six individuals were diagnosed in childhood (before age 18). 
Table 4. Diagnosis group (D), primary diagnoses, and specific illnesses of the patients included into the study.

\begin{tabular}{l|l|c|l}
\multicolumn{2}{c}{ PrIMARY DIAGNOSIS } & N \\
\hline D1 & Diabetes & 9 & Type 1, Type 2, insulin resistance \\
\hline D2 & Musculoskeletal diseases and trauma & 6 & $\begin{array}{l}\text { Rheumatoid arthritis, Bechterew disease, inflammatory arthropathy, } \\
\text { rheumatism, disc herniation, vertebral dislocation }\end{array}$ \\
\hline D3 & Digestive illnesses & 8 & $\begin{array}{l}\text { Acid reflux, intestinal polyps, gastric ulcers, functional disorders of the small } \\
\text { intestine, functional constipation, irritable bowel syndrome, ulcerative colitis, } \\
\text { Crohn's disease }\end{array}$ \\
\hline D4 & Nervous system diseases & 3 & Epilepsy, multiple sclerosis, Parkinson's disease
\end{tabular}

Patients in the diabetes diagnosis group (D1) were living with Type 1 diabetes ( $n=4)$, Type 2 diabetes ( $n=2)$ or insulin resistance $(n=3)$.

The second diagnosis group (D2) was comprised of patients with musculoskeletal diseases $(n=4)$ and musculoskeletal trauma that led to chronic conditions $(n=2)$. The third diagnosis group (D3) consisted of patients living with digestive illnesses: functional gastrointestinal disorders $(n=3)$, inflammatory bowel diseases $(n=2)$, and other gastrointestinal ailments $(n=3)$. The last diagnosis group (D4) comprised patients with nervous system diseases, namely: epilepsy, multiple sclerosis, and Parkinson's disease. Due to the small number of participants in D4, we will not be reporting on this diagnosis group in this study.

The following is an account of our results along the domains of etiology, push factors, and pull factors vis-à-vis rejecting conventional treatment. Rejecting conventional therapies a priori (forgoing) or a posteriori (discontinuing) was prevalent in both B and CAM groups, as even patients who use only conventional treatments had made decisions regarding employing one conventional treatment over another. We were interested in how rejecting conventional treatment was related to choice of therapy, as well as etiology and push/pull factors.

\section{Therapy choice and rejection of conventional treatment}

Therapy choice appeared on two levels in our coding process: in the creation of subsamples and in discourse codes. We created subsamples based on whether the patient employed conventional or non-conventional treatments regarding their primary diagnosis. This was coded as the patient belonging to the biomedicine (B) or the complementary and alternative medicine (CAM) subsample. Yet, patients regularly decide on whether they wish to adhere to or reject conventional treatments throughout the patient journey, not only concerning their primary diagnosis but also their comorbidities. To provide detailed insight on when and why patients rejected conventional treatments before trying them or after trying them, we coded transcripts with Reject prior and Reject post, respectively.

A priori rejection of conventional treatments occurred (no. of occurrences $=0$ ) in both subsamples $\left(O=78, O_{B}=35, O_{C A M}=43\right.$ ) and most prominently manifested as not taking prescribed medicine (or even filling a prescription) or not agreeing to undergo a 
pharmacological intervention (IDs: $2,4,5,7,10,11,15,16,18,19,21,22,26)$. Reasons for rejection included: the patient was against pharmaceuticals in general (or specifically against e.g., steroids or anti-depressants), was afraid of potential side-effects (e.g., weight gain), was fearful of becoming addicted, and did not want to switch to a new medicine. In some instances, diabetes patients refused to switch to an insulin pump (IDs 6, 16) because they were "not ready to change" and believed it would "feel foreign". Consumption of over-the-counter medication was also rejected in several cases in both subsamples (IDs 7, 8, 11, 15). Less frequently, patients rejected conventional treatments by not adhering to physician recommendations (e.g., diet, exercise) because "it's hard" to or because they "didn't take [the illness] seriously" (IDs 2, 3, 7, 20). Non-pharmacological interventions (e.g., surgery) were also rejected by patients (IDs $7,8,14,18,25$ ) because it could be postponed or avoided, and because the individual feared the procedure.

A posteriori rejection of conventional medicine occurred in both subsamples $\left(O=36, O_{B}=12, O_{C A M}=24\right)$. Pharmacological interventions were discontinued for various reasons (IDs: 3, 15, 18, 22, 24, 25, 26), such as avoiding severe side-effects or an "unnatural ingredient", the patient had heard negative things about the drug, wanted to decrease "the addiction" or felt they were "too young to be taking medicine regularly". Other explanations included: "I don't feel sick; I start taking it again when the pain comes back" and "I forgot [to take the medicine] and then never started again". Some CAM patients remarked that they went off conventional medicine because "it was interfering" with the CAM treatment. In lower frequencies, patients reported discontinuing physician-advised exercise because the classes stopped or they "didn't care enough" to attend; other patients went off their diet because it is "difficult to not be seduced" by various foods and seemed like "torture" (IDs 2, 7, 8, 11, 23).

Below is an account of code frequencies and co-occurrences. Quotations from patients are in quotation marks and italics; the speaker is identified with their interview ID (1-26) in parentheses. Code labels are in monospaced font and italics. All narratives were originally in Hungarian; quoted segments were translated into English by the lead author.

\section{Comparing Biomedicine and CAM users' narratives in terms of code frequency}

We performed a frequency count on our coded data; Figure 1 contains the total number of code occurrences (o) and their distribution among biomedicine and CAM user narratives for push/pull factor and etiology codes. Yet, because code occurrences may be influenced by the differing lengths of interview transcripts (number of utterances), we also computed normalized relative code frequencies for each code within B and CAM narratives. Frequencies were normalized by dividing the code counts per group (B and CAM) by the number of utterances, thus transforming the values to fall between zero and one. Figure 2 contains the normalized relative frequencies for push/pull factor and etiology codes. 


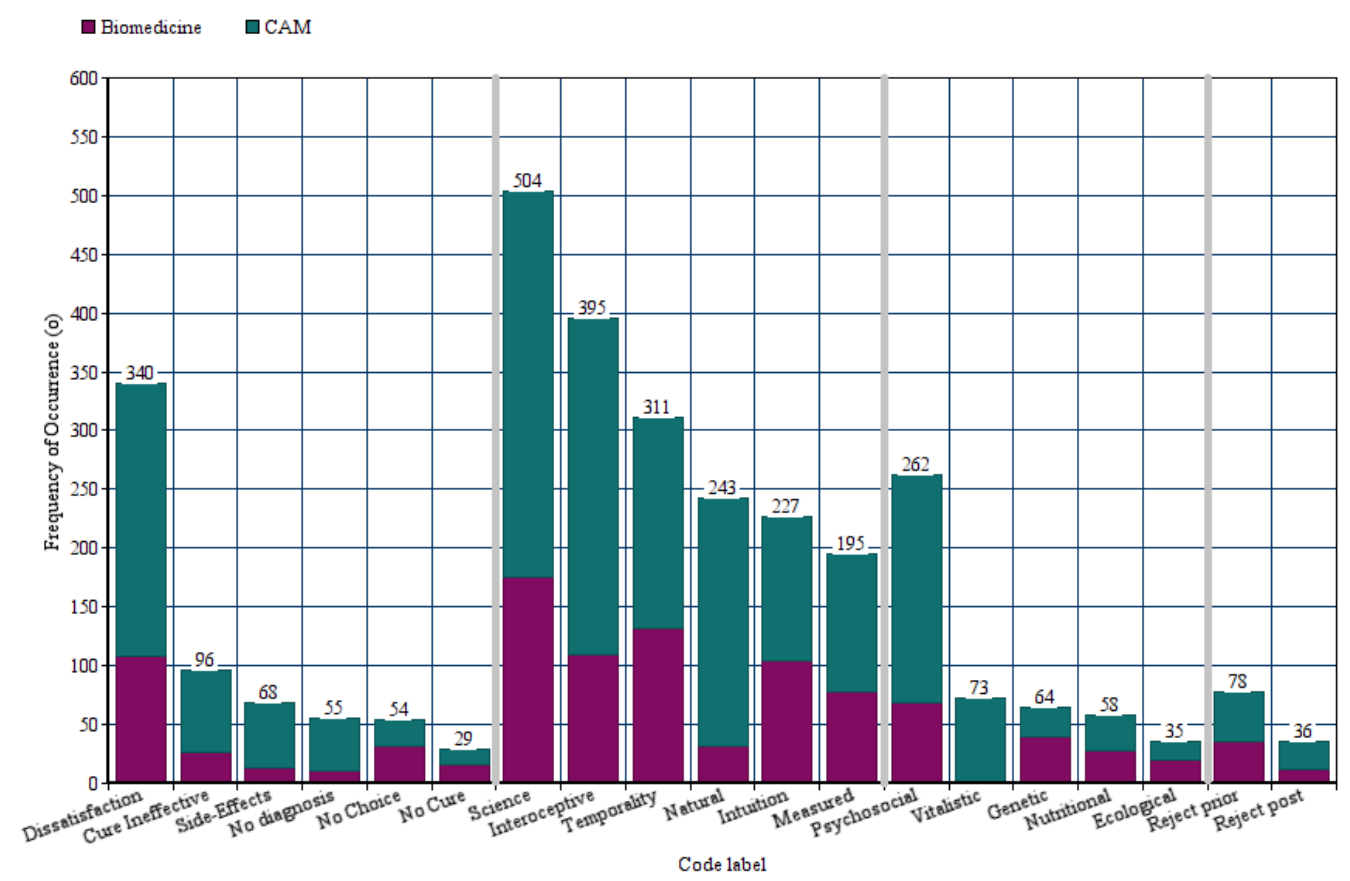

Figure 1: Code frequencies for Push/Pull factors and Etiology in Biomedicine (purple) and CAM users' (teal) narratives.

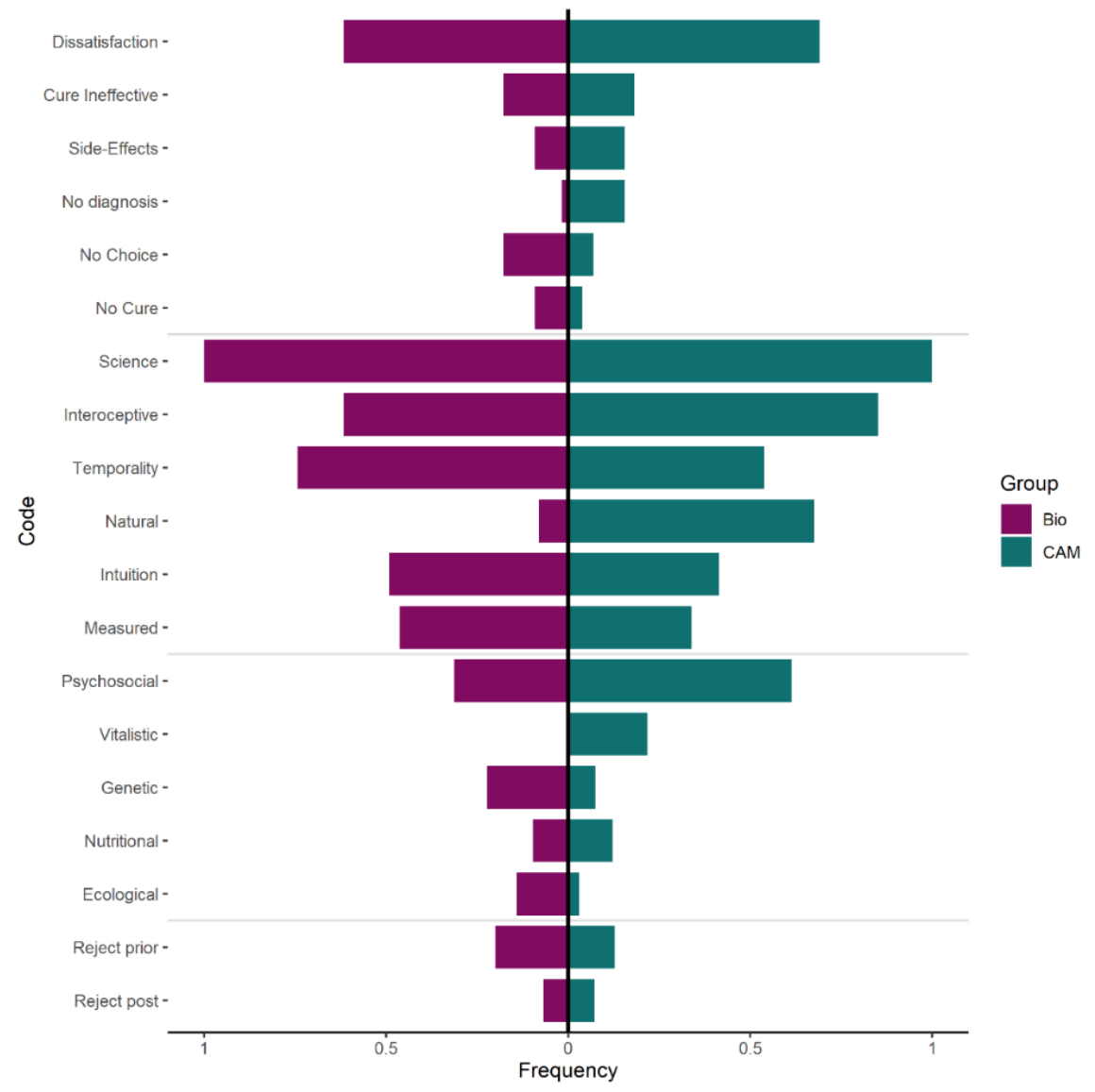

Figure 2: Scaled relative code frequencies for Push/Pull factors and Etiology in Biomedicine (purple) and CAM users' (teal) narratives. 
Among push factors, Dissatisfaction was the most frequent in coded data and it occurred more often in CAM narratives.

All other push factor codes, with the exception of No Choi ce had higher frequencies in CAM narratives than in the biomedicine group.

Yet, when viewing the normalized relative frequencies, it is clear that the differences in expressing Dissatisfaction were due to differing narrative lengths, as was the case with Cure Ineffective. Normalized relative code frequencies indicate that No diagnosis was mentioned more frequently in CAM interviews, and No Choice was emphasized more by biomedicine users. In terms of pull factor codes, Science was the most prevalent code in both groups, and all codes, save Natural, were similarly salient in biomedicine and CAM user narratives. Psychosocial was the most prominent etiology code in both groups, and CAM patients not only exhibited a higher number of occurrences, but also a higher relative frequency when accounting for varying number of utterances. Vitalistic was only referred to by CAM patients. Ecological and Genetic factors in the causation of disease were mostly commented on by users of biomedicine, and although Figure 1 shows that patients in both groups mentioned this code with a similar frequency, Figure 2 demonstrates that compared to the length of their narrative, Ecological was actually much more dominant in biomedicine group discourse.

Most critically, code frequencies show that both biomedicine and CAM users gave accounts of rejecting conventional treatments (either a priori or a posteriori) roughly to the same extent; thus, code counts alone cannot account for the difference in choice of therapy, nor can frequencies explain differences between the two groups regarding etiology and push/pull factors. Although relative code frequencies show us crucial features of the coded qualitative data, they do not lend insight into how codes interact within the narratives. Meaning is rarely constructed in isolation (e.g., one word or one code), and so code frequencies alone are not sufficient to understand the complexities of therapy choice. For this reason, we used ENA to model co-occurrences among codes (which accounts for frequency as well). The next section presents the ENA models developed to analyze the interaction between etiology and push/pull factor codes to explore patient motivations for rejecting conventional treatment. Each section contains results at the level of therapy choice (all biomedicine users versus all CAM users) and also at the level of diagnosis groups (D1-D3).

\section{NETWORK MODELS OF THE INTERACTION BETWEEN LAY ETIOLOGY AND REJECTING CONVENTIONAL TREATMENT}

\section{Comparing Biomedicine and CAM users in general}

Table 5 summarizes how etiology codes manifested in patient narratives. Most narrative fragments labeled with etiology codes differed greatly in our two subsamples (B and CAM). For biomedicine patients, the code Psychosocial etiologies denoted attention to self, good mental condition, and social support, which in their view contributed to adherence and self-management. As one biomedicine patient stated: "Exactly like if I don't do exercise, my spine starts to hurt, I need to keep a balance in being attentive 
to myself, and if I'm mentally well then I can pay attention to my things, my body" (ID 7). For users of CAM, Psychosocial signified the physiological effects of emotional trauma and stressors, and was highly associated with Vitalistic etiologies. The latter code manifested only among CAM patients and centered around concepts of energy: illness is impeded energy flow in the body and represents a conflict they need to "solve in this life" (the body "signals" in the form of illness), which they "chose to work on" and it is believed that if enough work is carried out within themselves, then the physical illness will heal. For example, several diabetes patients exhibited a symbolic interpretation of their illness: diabetes is "connected to love" and "receiving and giving", "I don't love myself enough and don't let others love me" (ID 20, 22).

The code Genetic in group B connoted that one is "born with a susceptibility" or "predisposed" to an illness; interviewees often even named a specific family member from whom they inherited their disease, and expressed a concern for passing it on to their future children. This code in CAM patient narratives most appeared as a vehicle for "carrying over 'material' from a past life" that the patient needs to "work with" (ID 18), such as inherited inter- or intrapersonal conflicts or behavioral patterns deemed destructive by the patient. One patient stated: "Illness always has an emotional cause, but because I was born this way [...] obviously something happened in a past life to cause this to me, because to this day, [conventional] medicine doesn't know why this sickness happens" (ID 25). The same patient remarked that psychological trauma to a family member can be "inherited" in the sense that the repercussions affect a descendant.

Nutritional etiological factors were typically regarded by CAM patients as inferior to emotional factors, who claimed that it does not matter what one eats if "your soul is continuously in upheaval" (ID 5). This indicates the presence of a unidirectional relationship between body and mind, where the former is subsumed by the primacy of the latter. ECOIogical etiology manifested as environmental factors (e.g., pollution) in group B, but were de-emphasized in CAM narratives with patients circling back to the responsibility of the individual in disease. One group B patient had an epigenetic approach, combining Ecological and Genetic factors: "Some people have a proven genetic predisposition, but even then, environmental factors and lifestyle play an important role in actually getting sick" (ID 17). 
Table 5. Etiology codes and their manifestations in both subsamples (B and CAM).

\begin{tabular}{|c|c|c|}
\hline CODE LABEL & GROUP & MANIFESTATION \\
\hline \multirow{7}{*}{ PSYCHOSOCIAL } & \multirow{4}{*}{ B } & Mental wellbeing that is a factor in adherence and perseverance in the "fight against a disease" \\
\hline & & "Emotional instability", which may interfere with commitment to a healthy lifestyle \\
\hline & & Attention, balance, social support, and reduced stress \\
\hline & & Neglecting oneself, fatigue, a reckless lifestyle, and too much work that exacerbates disease \\
\hline & \multirow{3}{*}{ CAM } & Emotional causes of physical disease \\
\hline & & Stress: diseases can only take hold if one's nervous system is "weak due to fatigue and anxiety" \\
\hline & & Effects of childhood trauma and anxiety \\
\hline \multirow{4}{*}{ VITALISTIC } & \multirow{4}{*}{ CAM } & Life events (e.g.: accidents, illnesses) are "created" by the individual so that they can "learn from it" \\
\hline & & "Energy" courses through the body; if there is a block in circulation it creates illness; the block must be "dissolved" in order to heal \\
\hline & & Illnesses have a greater meaning, they do not occur "by chance", and the individual must find the "real reason" for physical disease \\
\hline & & $\begin{array}{l}\text { Illness afflicting body parts (e.g.: hands) symbolize psychosocial problems (e.g.: "I do everything for everyone and what do I get in } \\
\text { return?") }\end{array}$ \\
\hline \multirow{5}{*}{ Genetic } & \multirow{2}{*}{ B } & "Susceptibility" or "predisposition" to an illness (which can be aggravated to some extent by a stressful lifestyle) \\
\hline & & Physiological irregularity that one is born with (e.g.: "my cardia does not close properly") \\
\hline & \multirow{3}{*}{ CAM } & Inherited inter- or intrapersonal conflicts or behavioral patterns from family or previous life \\
\hline & & Reincarnation and "brought material" the individual needs to "work on" \\
\hline & & "The stress-causing hormone" can be inherited \\
\hline \multirow{4}{*}{ NUTRITIONAL } & \multirow{2}{*}{ B } & Food as etiology (e.g. "bad diet", prolonged exposure to "bad ingredients", processed or fatty food, and sugar) \\
\hline & & Destructive habits (e.g. "going to extremes" in eating and not getting enough exercise) \\
\hline & \multirow{2}{*}{ CAM } & Non-regular eating habits, too much sugar consumption or "chemicals" in food and milk \\
\hline & & Inferior to emotional factors in illness causation \\
\hline \multirow{4}{*}{ ECOLOGICAL } & \multirow{3}{*}{ B } & $\begin{array}{l}\text { Environmental factors that exacerbate disease (e.g.: the weather, pollution, contaminated water) or worsen adherence to diet (e.g.: } \\
\text { social environment) }\end{array}$ \\
\hline & & "Living in a bad neighborhood" (i.e.: urbanized) \\
\hline & & "Environmental factors as discussed in conventional medicine" \\
\hline & CAM & $\begin{array}{l}\text { "Wanting to achieve too much" leads to overwork and an external locus of self-worth, yet all this conceptualized as "generated by } \\
\text { oneself" }\end{array}$ \\
\hline
\end{tabular}

There was a marked difference between groups biomedicine and CAM concerning etiology and rejecting conventional treatment. The most prominent code in both networks was Psychosocial; in group biomedicine this code was most connected to Genetic and Ecological, while in group CAM, it was strongly associated with Vitalistic and Nutritional. Biomedicine users made no connections to Vitalistic, but in the CAM group this code is strongly connected to Psychosocial. Biomedicine patients reported Psychosocial factors less and Genetic factors more frequently than CAM patients. In the CAM mean network, both Reject prior and Reject post exhibit strong connections to Psychosocial, in the biomedicine mean network the latter code retains a strong connection with Reject prior. Figure 3 shows the comparison plot and mean networks of our two subsamples. 


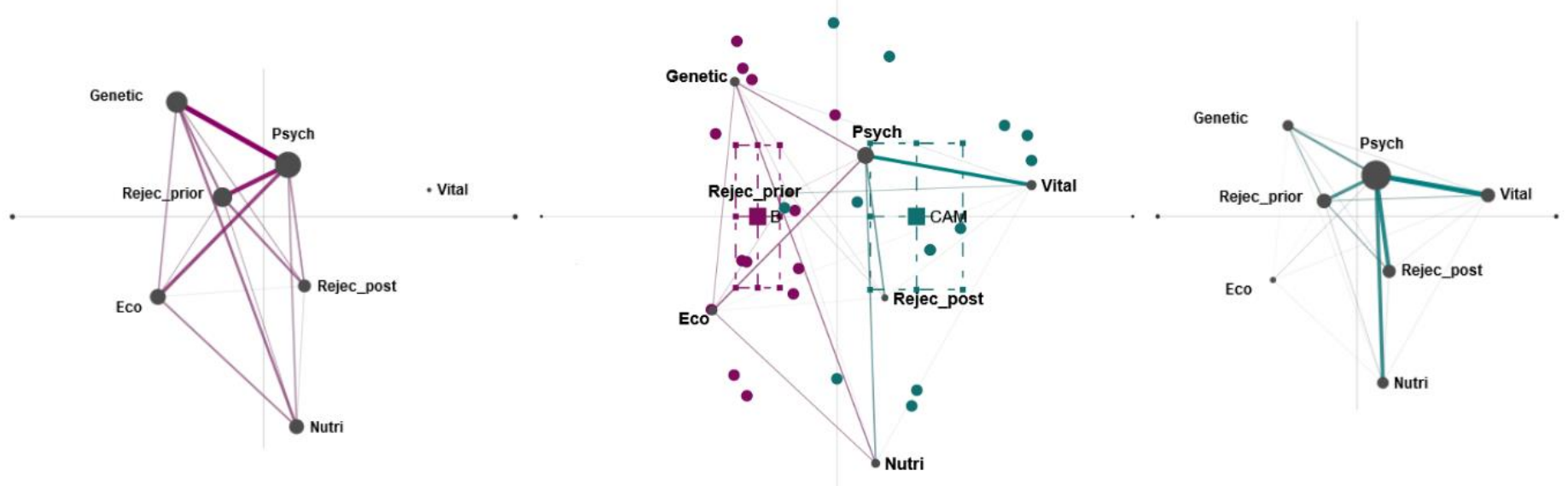

Figure 3. LEFT \& RIGHT: Mean epistemic networks for the biomedicine group (purple, left) and the CAM group (teal, right) showing the weighted structure of connections among etiology and rejection codes. The thickness and saturation of the edges (lines) indicate the relative frequency of co-occurrence between each pair of codes; the size of the nodes (black circles) indicates the relative frequency of each code within that group. CENTER: Difference graph showing the subtracted mean networks of the biomedicine group and the CAM group. The thickness and saturation of each line indicates the relative difference between the two groups: purple lines indicate connections with higher relative frequencies among biomedicine users, and teal lines indicate connections with higher relative frequencies among CAM users. The points show the network locations (ENA scores) of each biomedicine user (purple points) and CAM user (teal points). The colored squares are the mean network locations (mean ENA scores) of each group, and the dashed lines around the means represent the $95 \%$ confidence intervals on each dimension.

\section{Comparing Biomedicine and CAM users according to diagnosis}

Group biomedicine diabetes patients (D1) emphasized the connection between Genetic and Nutritional factors in illness etiology, while CAM patients exhibited those same connections supplemented with Vita list ic factors. The latter code was the least prominent in this group, compared to all other CAM networks. Psychosocial etiology had a strong connection with Reject prior in both groups. Group B patients living with musculoskeletal illnesses (D2) stressed connections among Psychosocial, Genetic and ECological factors; CAM narratives chiefly included the co-occurrence of Psychosocial and Vitalistic factors, this connection is the strongest in this network compared to all other CAM models. Psychosocial etiology retained a strong connection to rejecting conventional treatment in the CAM group, but only to Reject prior in group B. Biomedicine users in the digestive illnesses group (D3) made a strong connection between Psychosocial and Ecological factors in illness causation (the strongest in all models), and linked Genetic factors in as well. The connection between Psychosocial and Nutritional factors was the strongest in D3 CAM patient narratives, who de-emphasized Ecological factors compared to biomedicine users, but connected the code Vitalistic to Psychosocial with a relatively high frequency. Rejecting conventional treatment exhibits only weak connections to other codes in these networks. Figure 4 shows the mean networks for diagnosis groups D1-D3 in both subsamples. 
Mean epistemic networks for the three diagnosis groups (D1-3) in the biomedicine group (purple) and the CAM group (teal) regarding etiology codes and rejecting conventional treatment

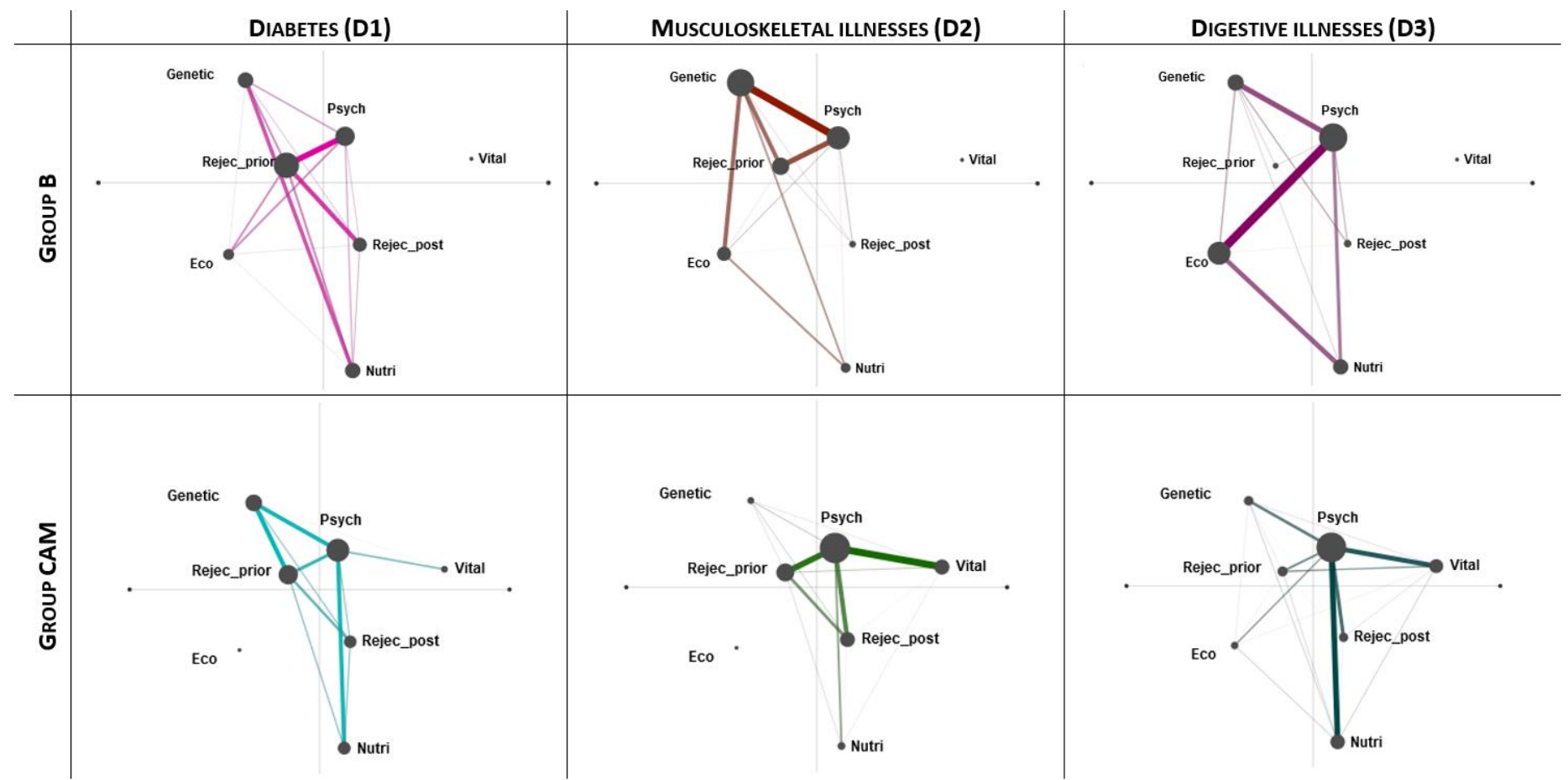

Figure 4. Mean etiology networks of the two subsamples: users of Biomedicine (top row) and CAM (bottom row) by diagnosis group (D1-D3, columns). Node size represents the relative frequency of codes within co-occurrences; edge thickness represents the connection strength (i.e., the relative frequency of co-occurrence) between two codes.

\section{NETWORK MODELS OF THE INTERACTION BETWEEN PUSH FACTORS AND REJECTING CONVENTIONAL TREATMENT}

\section{Comparing Biomedicine and CAM users in general}

Table 6 summarizes how push factor codes manifested in biomedicine and CAM user narratives. Most narrative fragments labeled with push factors were similar in the two groups, however, there were three key differences: (1) CAM patients expressed dissatisfaction regarding the over-prescription and over-use of pharmaceuticals, while biomedicine patients did not; (2) CAM patients interpreted side-effects of conventional medicine as detrimental effects on health that may outweigh their usefulness; and (3) CAM patients reported more instances of medically unexplained symptoms, which they sometimes attributed to severe side-effects from conventional treatments, but at other times to undiagnosed comorbidities: "I was always thinking about this and the problem consumed me [...] I wanted a diagnosis so that this calvary will end" (ID 4), as one patient reported. 
Table 6. Push factor codes and their manifestations in both subsamples (B and CAM).

\begin{tabular}{|c|c|c|}
\hline CODE LABEL & GRouP & MANIFESTATION \\
\hline \multirow{7}{*}{ DISSATISFACTION } & \multirow{5}{*}{ Both } & $\begin{array}{l}\text { Inadequate doctor-patient communication (perceived inability to ask questions, recount experiences, elaborate opinions, receive } \\
\text { empathy, sufficient/accurate information, lack of time in doctor-patient interaction) }\end{array}$ \\
\hline & & Conflicting opinions of various doctors \\
\hline & & "Malpractice" (misdiagnosis, mistreatment, or negligence as perceived by the patient) \\
\hline & & Perceived financial interest behind healing \\
\hline & & Systemic problems in healthcare \\
\hline & \multirow[b]{2}{*}{ CAM } & Over-prescription and over-use of pharmaceuticals \\
\hline & & $\begin{array}{l}\text { Juxtaposing conventional and CAM environments, the latter having a more helpful and open milieu, focusing on the "whole person } \\
\text { as opposed to merely the disease" }\end{array}$ \\
\hline \multirow{2}{*}{ CURE INEFFECTIVE } & \multirow{2}{*}{ Both } & Recurring symptoms, partial remission, complaints only partially treated, or intervention had no effect \\
\hline & & Even after diagnosis, having to try many interventions before symptoms subside \\
\hline \multirow{2}{*}{ SIDE-EFFECTS } & Both & Nausea, weight gain/loss, diminished liver function, itchy scalp, hair loss, fatigue, pain, rectal bleeding, etc. \\
\hline & CAM & Side-effects interpreted as detrimental effects of biomedicine (e.g., antibiotics, steroids, endoscopy) \\
\hline \multirow{3}{*}{ No DIAGNOSIS } & \multirow{2}{*}{ Both } & Lengthy patient journey before diagnosed: "Going from one doctor to another, [trying to] find out what the problem is" \\
\hline & & Presently experiencing a still undiagnosed comorbidity \\
\hline & CAM & Being alone in their interpretation of bodily sensations after conventional interventions \\
\hline \multirow{4}{*}{ No CHOICE } & \multirow{3}{*}{ Both } & Inability to change the circumstances of taking medicine, which medicine to take, or to undergo surgery \\
\hline & & Inability to control changes in body weight; having to pay close attention to diet, exercise, or sleep \\
\hline & & Having to admit to oneself that the disease is incurable \\
\hline & $\mathrm{B}$ & No viable alternatives to conventional medicine \\
\hline NO CURE & Both & No known cure for the disease (chronic illness or disease with no conventional cure) \\
\hline
\end{tabular}

As displayed in the ENA models, in both groups, the code Dissatisfaction co-occurred most frequently with other codes

in the corpus. The most prominent connections for Group B patients were among Dissatisfaction, No Choice, and Cure Ineffecti ve: "I did everything the doctor told me to, but [my body] didn't react the way it was supposed to ... then I really started to believe that this was my fault, that I was doing something wrong ... this was a difficult time, and then it became critical, so I switched doctors" (ID 1). This indicates that users of biomedicine may have felt dissatisfaction with a biomedical treatment, a doctor, or the healthcare system, yet still retained their exclusive use of conventional treatments. CAM patients, in contrast, tended to stress connections among Dissatisfaction, Side-Effects, Cure Ineffective, and No diagnosis: "I tried to help [the doctors] figure this [skin problem] out, but it's not connected to anything I eat or drink. So, one time I went to a dermatologist [...] and the doctor told me to stick my hand under water [to see if the red spots re-appear] and nothing happened. And it's always the same solution: they prescribe me some kind of steroid cream" (ID 11). This constellation of codes in the CAM group also co-occurred with rejection of conventional treatments; both Reject prior and Reject post had a strong connection to Dissatisfaction. Figure 5 shows the mean networks and difference graph of our two subsamples. 

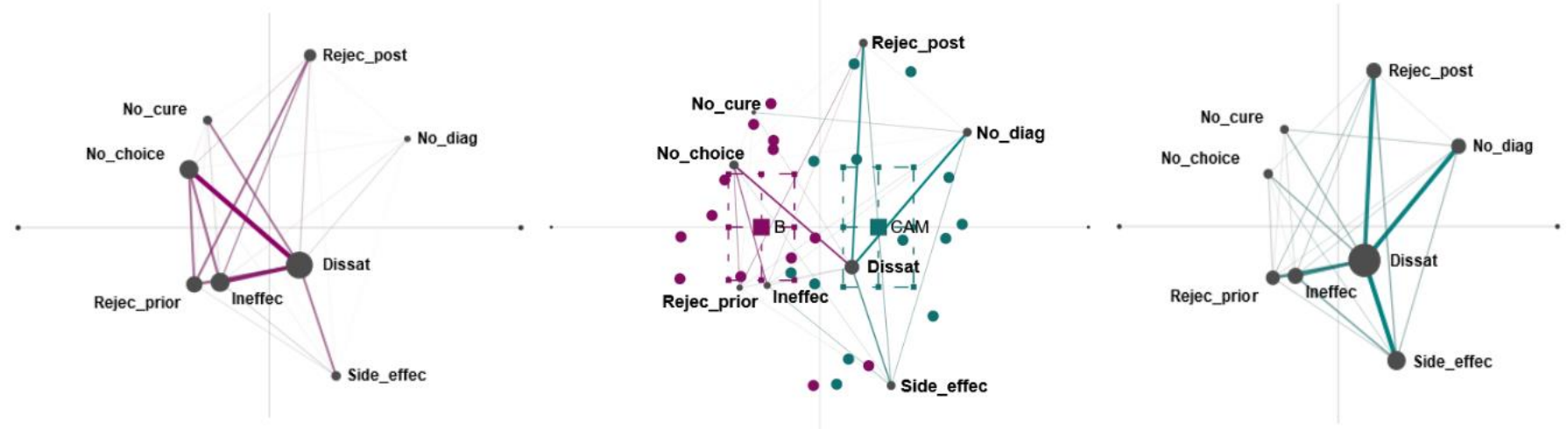

Figure 5. LEFT \& RIGHT: Mean epistemic networks for the biomedicine group (purple, left) and the CAM group (teal, right) showing the weighted structure of connections among push factor and rejection codes. The thickness and saturation of the edges (lines) indicate the relative frequency of co-occurrence between each pair of codes; the size of the nodes (black circles) indicates the relative frequency of each code within that group. CENTER: Difference graph showing the subtracted mean networks of the biomedicine group and the CAM group. The thickness and saturation of each line indicates the relative difference between the two groups: purple lines indicate connections with higher relative frequencies among biomedicine users, and teal lines indicate connections with higher relative frequencies among CAM users. The points show the network locations (ENA scores) of each biomedicine user (purple points) and CAM user (teal points). The colored squares are the mean network locations (mean ENA scores) of each group, and the dashed lines around the means represent the $95 \%$ confidence intervals on each dimension.

\section{Comparing Biomedicine and CAM users according to diagnosis}

The network of diabetes patients (D1) using only biomedicine exhibits a strong connection between Dissatisfaction and No Choice, indicating that patients spoke about their therapy choice as evident and having no other realistic alternatives. Although this was true for CAM diabetes patients as well, they emphasized inevitability less often and saw CAM as a possible supplementary therapy. One CAM patient employed non-conventional treatments as an alternative to conventional diabetes treatment. There is a strong connection between Dissatisfaction and Reject prior in the biomedicine group, illustrating times when diabetes patients were expressing hesitation to switch to insulin pump use. The biomedicine network also exhibits a strong connection between the two forms of rejecting conventional treatment; this indicates that biomedicine users described difficulties in first accepting and implementing an exercise regime, and adhering to that aspect of conventional treatment (e.g., ID 2). Another patient expressed their reservations about 3-Day Continuous Glucose Monitoring, explaining that it was ineffective and imprecise, and that they would not go through that process again (ID 16). While Dissatisfaction was only connected to Reject prior in the case of biomedicine users, in the CAM group, the former retained strong connections to both forms of rejecting conventional treatment. CAM patients also linked Dissatisfaction with Side-Effects when discussing their difficulties in dealing with diabetes, controlling insulin 
levels, and also having comorbidities treated; drugs and drug interactions produced side-effects, such as nausea and weight gain (ID $18,20)$.

Regarding musculoskeletal illness (D2), biomedicine users' network exhibits a strong connection between Dissatisfaction and Cure Ineffective, as well as No Choice and Cure Ineffective, indicating situations where the patient was dissatisfied with a certain (pharmacological) intervention they deemed ineffective (e.g., symptoms persisted or recurred) but felt they had no other alternative to treat the disease (ID 9, 19). CAM musculoskeletal patients living with rheumatoid and erosive arthritis emphasized Dissatisfaction and No Cure, characterizing the progressive nature of the chronic diseases, and the frequent need to try new medicines and other interventions that seem to be ineffective or too risky in their side-effects. As one CAM patient explained: "[At first they said] we don't know what the problem is, but it is incurable" (ID 26); she recalled being prescribed many pills and creams, none of which were effective, then finally she was prescribed a certain pharmaceutical, which, based on an internet search, she found was "given to severe cancer patients [...] a strong, aggressive drug" (ID 26). The long journey to diagnosis, the ineffective treatments, and the perceived risk of the "aggressiveness" and potential side-effects of a drug led several patients to use non-conventional medicine as an alternative to biomedicine. These were also the sentiments and experiences that led them to discontinue treatments and/or forgo interventions.

Biomedicine users in the digestive illnesses group (D3) emphasized the fact that there is no cure for their illness (e.g., ulcerative colitis), but their dissatisfaction was expressed concerning experiences prior to diagnosis. They did, however, stress that they had no choice in employing certain drugs and adhering to a strict diet; Dissatisfaction mostly referred to the physician not providing sufficient explanation or detail regarding their recommendations. Among digestive illness networks, the connection between Dissatisfaction and No diagnosis is stronger in the CAM group than in any other model, signifying instances of the patient failing to receive a diagnosis for their complaints prior to being diagnosed with their primary illness (with which they were included into the study) or for a comorbidity. Lengthy patient journeys during which the patient was either not diagnosed or successive treatment options were described as "trial and error" led to a priori or a posteriori rejection of conventional treatments. Figure 6 shows the mean networks for diagnosis groups D1-D3 in both subsamples. 
Mean epistemic networks for the three diagnosis groups (D1-3) in the biomedicine group (purple) and the CAM group (teal) regarding push factor codes and rejecting conventional treatment

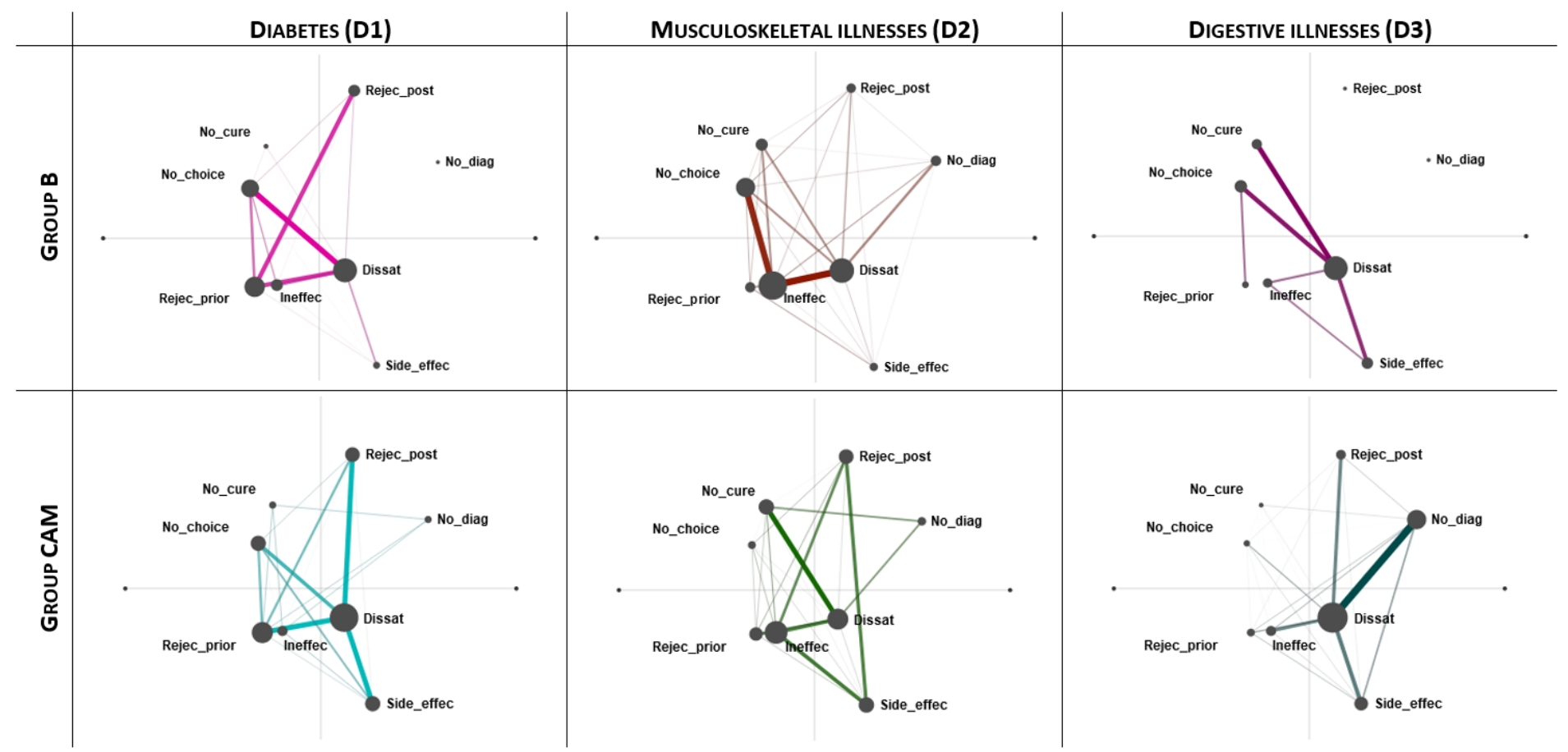

Figure 6. Mean push factor networks of the two subsamples: users of Biomedicine (top row) and CAM (bottom row) by diagnosis group (D1-D3, columns). Node size represents the relative frequency of codes within co-occurrences; edge thickness represents the connection strength (i.e., the relative frequency of co-occurrence) between two codes.

\section{NETWORK MODELS OF THE INTERACTION BETWEEN PULL FACTORS AND REJECTING CONVENTIONAL TREATMENT}

\section{Comparing Biomedicine and CAM users in general}

Table 7 summarizes how pull factor codes manifested in biomedicine and CAM user narratives. The biggest difference between the two groups was that CAM patients expressed the code Natural nearly seven times more often than did biomedicine patients; these manifestations referred to products (e.g., dietary supplements, vitamins), CAM modalities (e.g., acupuncture, phytotherapy), and self-help techniques (e.g., meditation, yoga). Natural phenomena were employed for controlling blood sugar, aiding circulation, minor diseases such as colds, as well as with skin problems and loss of hair, digestive issues, and strengthening the immune system. Furthermore, while both biomedicine and CAM patients employed Intuition to make decisions regarding specific interventions, CAM patients interpreted their intuition as a "sign" to use CAM. 
Table 7. Pull factor codes and their manifestations in both subsamples (B and CAM).

\begin{tabular}{|c|c|c|}
\hline CODE LABEL & GROUP & MANIFESTATION \\
\hline \multirow{5}{*}{ SCIENCE } & \multirow{5}{*}{ Both } & Using scientific studies or results to support a claim or prove the efficacy of a treatment \\
\hline & & Expressing hope that science will develop new medical technologies or a cure for illness \\
\hline & & Stating a need for science to interpret one's health status or biomedicine to diagnose a disease \\
\hline & & $\begin{array}{l}\text { Using high-level medical terms (e.g., about a comorbidity: "The thing with Hashimoto's is, you can't set the level of L-Thyroxin like } \\
\text { you can with hypothyreosis or hyperthyreosis") }\end{array}$ \\
\hline & & Asserting an explicit belief in science or conventional medicine \\
\hline \multirow{4}{*}{ INTEROCEPTIVE } & \multirow{4}{*}{ Both } & Referring to bodily sensations to determine state of health/illness (e.g., blood sugar level, general condition) \\
\hline & & Referring to bodily sensations to determine the "good"/"bad" effects of intervention \\
\hline & & Referring to bodily sensations to determine the effects of lifestyle decisions (e.g., diet, regular exercise) \\
\hline & & Referring to bodily sensations make health-related decisions \\
\hline \multirow{4}{*}{ TEMPORALITY } & \multirow{4}{*}{ Both } & Using time to support the (in)efficacy of a conventional treatment ("The treatment works fast") \\
\hline & & Using time to experientially test or monitor efficacy ("We should see results in a month or two") \\
\hline & & Using time to explain modus operandi ("It is absorbed by the body over 48 hours, not 24 ") \\
\hline & & Using time as proof of healing or that condition has stabilized \\
\hline \multirow{4}{*}{ NATURAL } & \multirow{4}{*}{ CAM } & Products (e.g., dietary supplement, cannabis, cremes, shampoo, vitamins, fruits and vegetables) \\
\hline & & CAM modalities/services (e.g., bioresonance, naturopathy, acupuncture, phytotherapy, homeopathy) \\
\hline & & Self-help techniques ("Eastern" forms of exercise, yoga, meditation) \\
\hline & & $\begin{array}{l}\text { Synonyms: "from nature", "medicinal plants", "ancient", having "no chemicals", "no side-effects", and not being a pharmaceutica } \\
\text { or an addictive substance }\end{array}$ \\
\hline \multirow{2}{*}{ INTUITION } & Both & Basing decisions or opinions on: “just knew it”, "just felt it”, “knew from experience” \\
\hline & CAM & Basing decisions or opinions on: being "partial" to something or considering it "a sign" \\
\hline \multirow[b]{2}{*}{ MEASURED } & \multirow[b]{2}{*}{ Both } & Diagnostic testing or measurement (e.g., ultrasound, blood work, biopsy) \\
\hline & & $\begin{array}{l}\text { Referring to biomedical parameters (e.g., blood sugar, blood pressure, hormone levels, weight) in order to diagnose, screen, or } \\
\text { monitor health status or the progression of disease }\end{array}$ \\
\hline
\end{tabular}

There was no marked difference between groups biomedicine and CAM concerning pull factors and rejecting conventional treatment. The most prominent code in the entire narrative corpus was Science: the patient referring to science and medicine, for example, as a trusted source of information, a preference, or a justification for a choice. In terms of frequency within co-occurrences, Science was followed by Interoceptive and Temporality in both groups. The key difference between biomedicine and CAM user narratives was that CAM patients mentioned Natural more frequently and it co-occurred more often with other codes. As one CAM diabetes patient remarked: "I read about this dietary supplement [...] they don't even sell it in Hungary [...] but my brother lives in England and I asked him to send some to me [...] and I've been taking it for 6 days now and let me tell you, I feel almost new! It has no side-effects, no chemicals, so it's made from completely natural ingredients" (ID 18). Thus, CAM patients relied heavily on Interoceptive methods to determine efficacy and justify choosing Natural treatments. Yet, rejecting conventional therapy is not prominent in either mean network. Figure 7 shows the difference graph and mean networks of the CAM and Biomedicine groups for the interaction between pull factors and rejecting conventional treatment. 

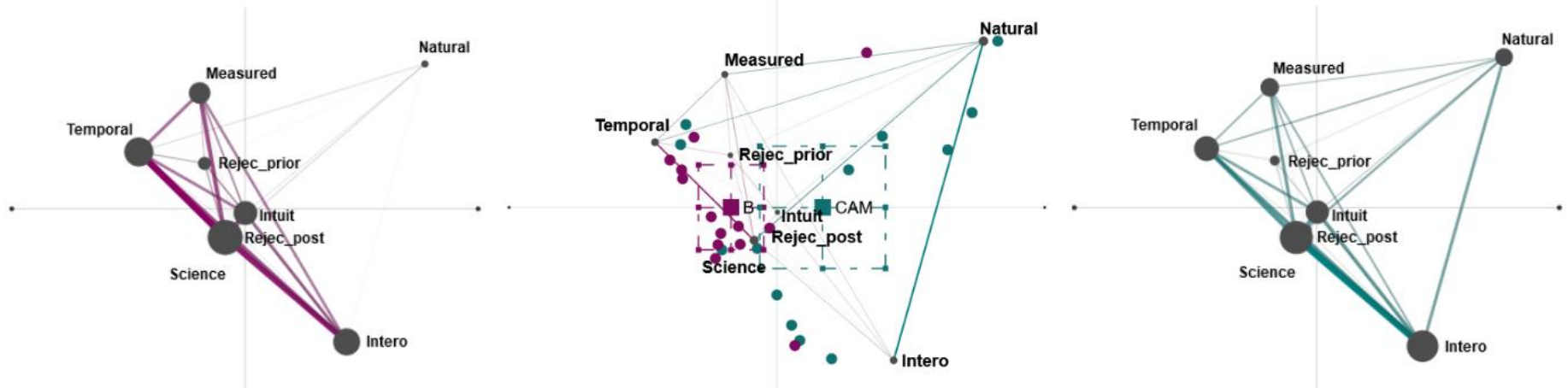

Figure 7. LEFT \& RIGHT: Mean epistemic networks for the biomedicine group (purple, left) and the CAM group (teal, right) showing the weighted structure of connections among pull factor and rejection codes. The thickness and saturation of the edges (lines) indicate the relative frequency of co-occurrence between each pair of codes; the size of the nodes (black circles) indicates the relative frequency of each code within that group. CENTER: Difference graph showing the subtracted mean networks of the biomedicine group and the CAM group. The thickness and saturation of each line indicates the relative difference between the two groups: purple lines indicate connections with higher relative frequencies among biomedicine users, and teal lines indicate connections with higher relative frequencies among CAM users. The points show the network locations (ENA scores) of each biomedicine user (purple points) and CAM user (teal points). The colored squares are the mean network locations (mean ENA scores) of each group, and the dashed lines around the means represent the $95 \%$ confidence intervals on each dimension.

\section{Comparing Biomedicine and CAM users according to diagnosis}

CAM networks exhibited no major differences. The only markedly different biomedicine model was that of the digestive illnesses group containing connections to Natural. Diabetes (D1) networks are similar to each other and their mean networks, with strong connections among Science, Temporality, Interoceptive, Intuition and Measured. The only marked difference between biomedicine and CAM networks is that where CAM patients' narratives manifested the code Natural, biomedicine patients' did not. Musculoskeletal illness (D2) networks are similar to each other and their mean networks, with strong connections among Science, Temporality, Interoceptive. As with D1, musculoskeletal CAM patients reported the code Natural and biomedicine patients did not. In the digestive illness (D3) CAM network the code Natural is the least prominent (compared to all other CAM networks and the mean CAM network), but it appeared in the biomedicine network, with connections to most pull factor codes. The code Measured is the least salient in the D3 CAM network, compared to all other networks. Rejecting conventional therapies does not have strong connections in either diagnosis group model. Figure 8 shows the mean networks for diagnosis groups D1-D3 in both subsamples. 
Mean epistemic networks for the three diagnosis groups (D1-3) in the biomedicine group (purple) and the CAM group (teal) regarding pull factor codes and rejecting conventional treatment

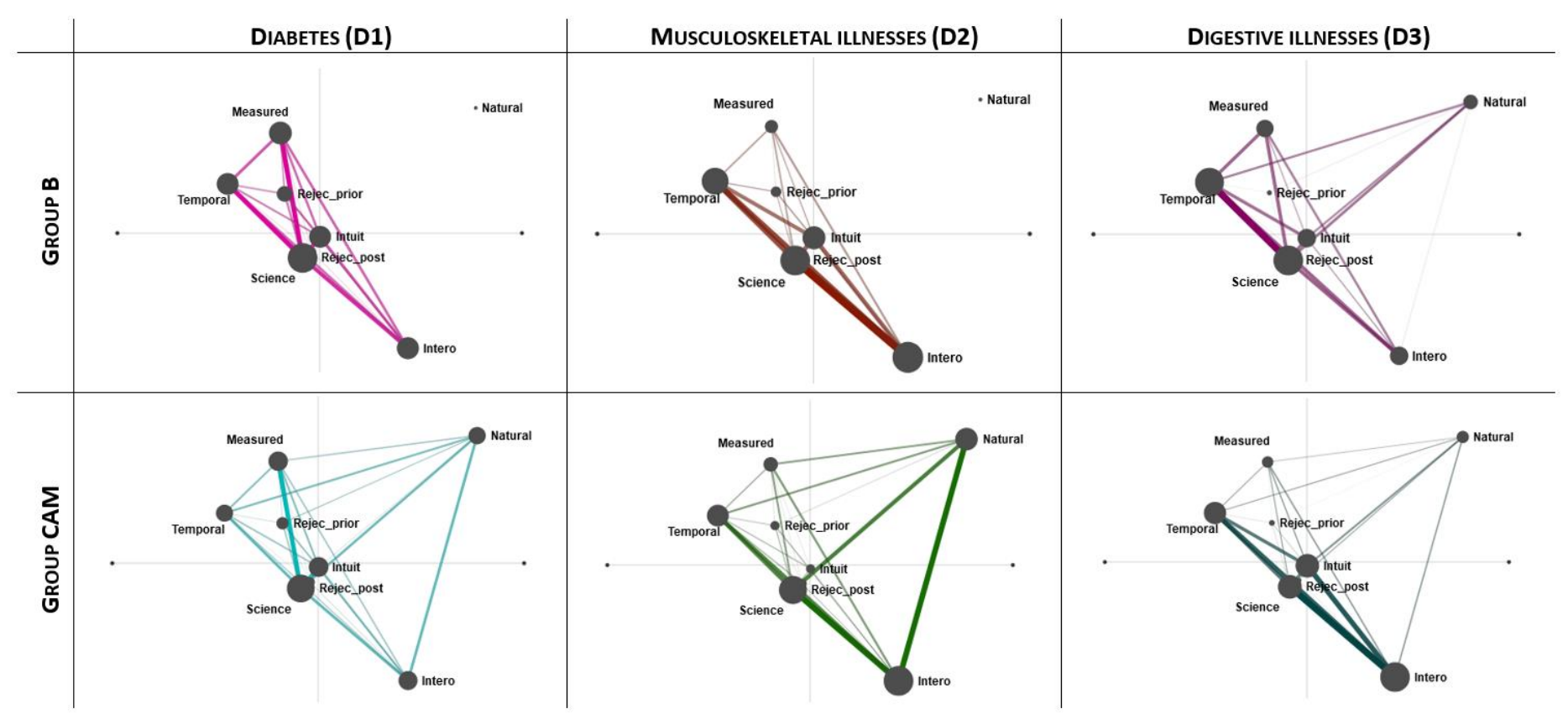

Figure 8. Mean pull factor networks of the two subsamples: users of Biomedicine (top row) and CAM (bottom row) by diagnosis group (D1-D3, columns). Node size represents the relative frequency of codes within co-occurrences; edge thickness represents the connection strength (i.e., the relative frequency of co-occurrence) between two codes.

\section{DISCUSSION}

We investigated the connection between rejecting conventional treatment (forgoing or discontinuing an intervention) and therapy choice (biomedicine or CAM use) with regards to: 1) lay etiology, 2) push factors, and 3) pull factors. We conducted semistructured interviews with users of only biomedicine and users of CAM, and analyzed the differences across patients' narratives. Our analysis began by inspecting code frequencies according to group, but these frequencies were not informative in many instances, as transcripts varied in length, which greatly influenced code counts. For this reason, we normalized the frequencies, which explained some differences between the two subsamples, but did not capture how patients integrated different elements of decision-making within their narratives of therapeutic choice. To model this integration, we analyzed code co-occurrences using ENA.

We found a marked difference between our two subsamples (all biomedicine users versus all CAM users) in the domain of push factors. Dissatisfaction was the most frequent push factor code in both subsamples; thus, dissatisfaction alone does not explain differences in choice of therapy. ENA models show that while the co-occurrence of Dissatisfaction, No Choice, and Cure Ineffective is strong in group B, it does not explain why patients turned to CAM. In the CAM models, Dissatisfaction, SideEffects, Cure Ineffective, and No diagnosis all exhibit strong connections to each other. This indicates that CAM patients' 
choice of therapy was greatly influenced by dissatisfaction stemming from experiencing a) a lengthy patient journey before being diagnosed, b) difficulties in treating comorbidities, c) medically unexplained symptoms, and d) side-effects from conventional treatment accredited to inefficacy. The network models indicate that it is not the individual occurrence but the co-occurrence of the aforementioned four codes that together led to rejecting conventional treatments and using CAM. These results imply that despite literature suggesting that pull factors outweigh push factors in patient decision-making (Arthur et al., 2012), the latter's cumulative effect throughout the patient journey might have a stronger influence on choice of therapy than previously theorized.

Pull factors yielded no large differences between our two subsamples. Science and Interoceptive were the two most prominent codes in mean networks on both the subsample and the diagnosis group level. This indicates that scientific evidence and worldview are on par with subjective experience among all patients. Some studies (e.g., Köteles et al., 2012) have asserted that Western CAM patients are more likely to dismiss evidence-based findings and retain an anti-scientific worldview, but our results suggest that acceptance of science is shared by both subsamples, and a subscription to "natural" products/services is a more crucial preference that distinguishes them. Manifestations of the code Natural connoted the largest difference between the two subsamples regarding pull factors. CAM patients not only mentioned Natural more frequently, but it also co-occurred with other dispositions more frequently (such as employing interoception to determine efficacy and justify therapeutic choices). Other scholars have examined the conceptualization of "natural therapies" by CAM users: therapies usually equated with herbal, folk or traditional medicine that seem like harmless therapies drawing on the body’s “self-healing” mechanisms (Zörgő \& Olivas Hernández, 2018). With a preference for the "natural", pharmaceuticals may be seen as "chemicals" to be avoided (Connor, 2004; McClean \& Shaw, 2005), which can in turn lead to a significant decrease in adherence to prescribed medication (Alfian et al., 2016). Congruently, a preference for the "natural" may be a reaction to the necessity of consuming pharmaceuticals on a daily basis as captured in the perception of drug "over-use" and "over-prescription" in our study. While "natural" therapies are usually seen as "benign" and safe (Tascilar et al., 2006), some conventional treatments - despite being life-saving interventions in many cases - may be perceived as "toxic" (Arthur et al., 2012; Citrin et al., 2012). Regardless, a preference for the Natural alone did not explain therapy choice (there were no major differences between mean networks), and it did not retain a strong connection to rejecting conventional treatments (as a discourse code within the networks).

We found a marked difference between our two subsamples regarding lay etiology. The most prominent code, Psychosocial, exhibited strong connections to rejecting conventional therapy in all models with the exception of the digestive illnesses group. Thus, etiology was a critical determining factor in both therapy choice and in rejecting conventional therapy. In general, Psychosocial etiology was most strongly connected with Vitalistic in the CAM groups, while among biomedicine users, it was 
associated with Genetic and Ecological. It is important to note that Psychosocial manifested in different ways. For biomedicine users in all subgroups, it connoted attention to self, (non)adherence to a healthy lifestyle, and social support; for CAM users it included the effects of childhood emotional trauma, anxiety concerning current stressors, and a physical manifestation of these. Biomedicine patients retained no connection to vitalism, but CAM patients in all diagnosis groups exhibited connections between psychosocial factors and vitalism. Patients associated vitalism with teleology and illness symbolism.

Although there are variations in vitalistic convictions, there is a general consensus that in order to experience one's sacred "Self" (as opposed to the profane "Ego"), one must "learn" and achieve "personal growth" via hardships like somatic illness (Reddy, 2002). Through a belief that "learning" can occur via life events, vitalism may also be linked to teleology - explaining phenomena by a grander "purpose" they serve, rather than their cause. These beliefs, in turn, may be associated with body or illness symbolism, serving as a system for interpreting the somatic "signs sent by the Self" (Coulter \& Willis, 2004; Heelas, 2013; Thompson, 2003). According to our results, vitalism, as a worldview, influenced the interpretation of all other etiological factors: in a vitalistic framework, genetic etiology manifested as reincarnation, psychosocial etiology as emotional trauma causing disease, and so on. This is because vitalism can function as a "prism" through which somatic events, social interactions, and ontology in general can be viewed (Reddy, 2002), and thus it may exert a strong effect on decision-making.

\section{CONCLUSIONS}

Our results suggest that push factors may have a significant role in decision-making regarding choice of therapy, but not as individual factors, such as dissatisfaction with conventional medicine or experiencing side-effects. Push factors exert a strong effect on therapy choice as a constellation of phenomena, which results in the rejection of conventional treatment. Among pull factors, the most crucial was the preference for natural treatments, which not only governed treatment choices but also the justification of using them and determining therapeutic efficacy. There was a marked difference in our subsamples concerning lay etiology: subscribing to vitalism affected the patient's interpretation of other etiological factors and their own decisions regarding viable treatment options. Our analysis moved beyond "code-and-count" techniques to model the complex interplay among codes manifesting in patient narratives to more accurately capture how patients describe their therapeutic decisions.

\section{LIMITATIONS, STRENGTHS, AND CONSIDERATIONS FOR FURTHER INQUIRY}

Our study had several limitations. Most crucially, our sampling was not random, and thus our analysis may only reflect the characteristics of the patients who opted into the study. Additionally, by continuing data collection we intend to further split the CAM 
group into complementary (parallel to biomedicine) and alternative (instead of biomedicine) users of non-conventional medicine. This is an important distinction to make, especially concerning diseases where rejecting conventional treatment threatens the patient's life. Not only therapy choice can be investigated in a more detailed grouping, but illness as well. Our current diagnosis groups may not be sufficiently specified, as, for example, a patient with life-threatening Crohn's disease and ulcerative colitis is in the same subgroup as a patient with acid reflux. On a similar note, the current ICD-specific categorization of disease could be augmented or modified to a cross-categorical nosology, for example, autoimmune versus non-autoimmune, chronic versus acute, curable versus incurable.

Aspects of our discourse segmentation influenced code frequency: upon automated designation of utterances, line breaks sometimes occurred within a sentence, and our decision was to code both sentence fragments with the code instead of arbitrarily choosing one fragment. Furthermore, segmentation choices affected the frequency of code co-occurrence as well, namely, in considering an entire interview transcript as a meaningful narrative segment, all codes within an interview could potentially co-occur with each other. Due to the chosen manner of accumulating code co-occurrences ("whole conversation"), the location of codes within the transcript and relative to each other did not influence the strength of their connections. Varying model parameters have been shown to have less of an effect on sample-level analyses, but individual networks may differ substantially (A. Ruis et al., 2019; Zörgő et al., 2021).

Our coding could be more specific to differentiate among the primary illness and its comorbidities in patient narratives, which may be pertinent to all codes included in this study. Similarly, our coding could expand into a temporal dimension, indicating whether the patient is expressing, e.g., dissatisfaction about current or past health-related events, or indicating that they tried an "ineffective cure" before they were properly diagnosed or subsequent to that. Finally, our models only contain the etiological and push/pull factors that we coded for; there may be other constellations of such factors that are not included in the networks.

The main strength of our analytical approach is that, by generating network models of code co-occurrences, we were able to move beyond reporting relative code frequencies and scrutinizing codes in isolation. Through an analytical process that unified qualitative and quantitative approaches, we could visually inspect network models and compare those to a hermeneutic interpretation of the coded texts. We were able to formulate statements about users of biomedicine and users of CAM in our sample, but we could also explore networks and narratives in various subgroups based on illness. This process enabled us to model the complexity of narratives and gain a more in-depth understanding of therapy choice. Furthermore, because our entire analytical process was performed in a transparent and machine-readable manner, it increased confirmability and enabled more scrutiny and reflection.

Because this was an exploratory analysis, however, we did not report statistical comparisons between the groups in the network models; that is, we did not conduct null hypothesis significance tests. Because the effects of lay etiology, push factors, and 
pull factors were explored using different codes, and thus the models have different parameters, it also is difficult to compare those models to one another. Comparison of models with different parameters is often done by using ENA scores as predictors in regression equations (see, e.g., Swiecki et al., 2019), and in future work, we will investigate how best to conduct such a comparison.

\section{LIST OF ABBREVIATIONS}

$\mathrm{CAM}=$ Complementary and Alternative Medicine (subsample)

$\mathrm{B}=$ Biomedicine (subsample)

ENA = Epistemic Network Analysis

$D=$ Diagnosis group

ROCK = Reproducible Open Coding Kit

UID = Utterance Identifier

$M R=$ Means Rotation

SVD = Singular Value Decomposition

$\mathrm{O}=$ Number of code Occurrences

ID = Patient/Transcript identifier

\section{DeCLARATIONS}

\section{Ethics approval and consent to participate}

Informed consent was obtained from all participants. The ethics permit was issued by Semmelweis University Regional and Institutional Committee of Science and Research Ethics (SE RKEB: 226/2018).

\section{Consent for publication}

Not applicable.

\section{Availability of data and materials}

Our entire process and all generated materials are openly available in our public repository (available at: https://osf.io/7sm5n/). Due to its sensitive nature, a certain proportion of our data was masked and only part of it was made open.

\section{Competing interests}

The authors declare that they have no competing interests.

\section{Funding}

No funding was received for this study.

\section{Authors' contributions}

Author contributions are based on CRediT (Contributor Roles Taxonomy). SZ: Conceptualization, Data Curation, Formal Analysis, Investigation, Methodology, Project Administration, Supervision, Visualization, Writing - original draft, review and editing; GJYP: Conceptualization, Data Curation, Methodology, Software, Validation, Visualization, Writing - review and editing; KCV, AG, and AJ: Data Curation, Formal Analysis, Investigation, Writing - review and editing; ARR: Supervision, Validation, Visualization, Writing review and editing.

\section{Acknowledgements}

The authors wish to thank participants of the study for their trust and contributions. 


\section{Works Cited}

Alfian, S. D., Sukandar, H., Arisanti, N., \& Abdulah, R. (2016). Complementary and alternative medicine use decreases adherence to prescribed medication in diabetes patients. Annals of Tropical Medicine and Public Health, 9(3), 174.

Arnault, D. (2009). Cultural Determinants of Help Seeking: A model for research and practice. Res Theory Nurs Pract., 23(4), $259-278$.

Arthur, K., Belliard, J., Hardin, S., Knecht, K., Chen, C., \& Montgomery, S. (2012). Practices, Attitudes, and Beliefs associated with Complementary and Alternative Medicine (CAM) use among Cancer Patients. Integr Cancer Ther., 11(3), $232-242$.

Astin, J. (1998). Why patients use alternative medicine: Results of a national study. JAMA, 279, 1548-1553.

Bishop, F., Yardley, L., \& Lewith, G. (2007). A systematic review of beliefs involved in the use of complementary and alternative medicine. J Health Psychol., 12(6), 851-867.

Boon, H., Brown, J. B., Gavin, A., \& Westlake, K. (2003). Men with Prostate Cancer: Making Decisions about Complementary/Alternative Medicine. Medical Decision Making, 23(6).

Bowman, D., Swiecki, Z., Zhiqiang, C., Wang, Y., Eagan, B., Linderoth, J., \& David Williamson, S. (2021). The Mathematical Foundations of Epistemic Network Analysis. In Advances in Quantitative Ethnography. Communications in Computer and Information Science Series. (Eds. Ruis AR and Lee, SB., Vol. 1312, pp. 91-105). Springer Nature.

Buckingham Shum, Simon, Echeverria, Vanessa, \& Martinez-Maldonado, Roberto. (2019). The Multimodal Matrix as a Quantitative Ethnography Methodology. In B. Eagan, M. Misfeldt, \& A. Siebert-Evenstone (Eds.), Advances in Quantitative Ethnography: First International Conference, ICQE 2019, Madison, WI, USA, October 20-22, 2019, Proceedings (pp. 26-40). International Society for Quantitative Ethnography.

Cartwright, T., \& Torr, R. (2005). Making sense of illness: The experiences of users of complementary medicine. J Health Psychol., 10(4), 559-572.

Citrin, D. L., Bloom, D. L., Grutsch, J. F., Mortensen, S. J., \& Lis, C. G. (2012). Beliefs and perceptions of women with newly diagnosed breast cancer who refused conventional treatment in favor of alternative therapies. The Oncologist, 17(5), 607-612.

Connor, L. (2004). Relief, risk and renewal: Mixed therapy regimens in an Australian suburb. Soc Sci Med., 59(8), 1695-1705.

Coulter, I., \& Willis, E. (2004). The rise and rise of complementary and alternative medicine: A sociological perspective. Med J Aust., 180(11), 587-589.

Eardley, S., Bishop, F., Prescott, P., Cardini, F., Brinkhaus, B., Santos-Rey, K., Vas, J., von Ammon, K., Hegyi, G., Dragan, S., Uehleke, B., Fønnebø, V., \& Lewith, G. (2012). A systematic literature review of complementary and alternative medicine prevalence in EU. Forsch Komplementmed., 19(Supp/ 2), 18-28. 
Frank, A. (1993). The Rhetoric of Self-Change: Illness Experience as Narrative. The Sociological Quarterly, 34(1), 39-52.

Furnham, A., \& Vincent, C. (2000). Reasons for using CAM. In Complementary and alternative medicine: Challenge and change.

(Kelner M, Wellman B, Pescosolido B, Saks M (szerk.), pp. 61-78). Harwood Academic Publishers.

Geertz, C. (1973). The Interpretation Of Cultures. Basic Books.

Goldstein, M. (2002). The Emerging Socioeconomic and Political Support for Alternative Medicine in the United States. Annals of the American Academy of Political and Social Science, 583(1), 44-63.

Harris, P. (2012). Prevalence of complementary and alternative medicine (CAM) use by the general population: A systematic review and update. Int J Clin Pract., 66(10), 924-939.

Heelas, P. (2013). On transgressing the secular: Spiritualities of life, idealism, vitalism. In New age spirituality: Rethinking religion. (SJ Sutcliffe, IS Gilhus (szerk.), pp. 66-83). Acumen Publishing.

Hunt, K., Coelho, H., Wider, B., Perry, R., Hung, S., Terry, R., \& Ernst, E. (2010). Complementary and alternative medicine use in England: Results from a national survey. Int J Clin Pract., 64(11), 1496-1502.

Keene, M. R., Heslop, I. M., Sabesan, S. S., \& Glass, B. D. (2019). Complementary and alternative medicine use in cancer: A systematic review. Complementary Therapies in Clinical Practice, 35, 33-47.

Knoll, A. (2004). The reawakening of complementary and alternative medicine at the turn of the twenty-first century: Filling the void in conventional biomedicine. J Contemp Health Law Policy, 20(2), 329-366.

Köteles, F., Bárány, E., Varsányi, P., \& Bárdos, G. (2012). Are modern health worries associated with somatosensory amplification, environmental attribution style, and commitment to complementary and alternative medicine? Scandinavian Journal of Psychology, 53, 144-149.

Maskarinec, G., Gotay, C., Tatsumura, Y., Shumay, D., \& Kakai, H. (2001). Perceived cancer causes: Use of complementary and alternative therapy. Cancer Pract., 9(4), 183-190.

McClean, S., \& Shaw, A. (2005). From Schism to Continuum? The Problematic Relationship Between Expert and Lay Knowledge-An Exploratory Conceptual Synthesis of Two Qualitative Studies. Qualitative Health Research, 15(6), 729-749.

Reddy, S. (2002). Asian Medicine in America: The Ayurvedic Case. Annals of the American Academy of Political and Social Science., $583,97-121$.

Ruis, A. R., Rosser, A. A., Quandt-Walle, C., Nathwani, J. N., Shaffer, D. W., \& Pugh, C. M. (2018). The hands and head of a surgeon: Modeling operative competency with multimodal epistemic network analysis. American Journal of Surgery, 216(5), 835840. 
Ruis, A., Siebert-Evenstone, A. L., Pozen, R., Eagan, B. R., \& Williamson Shaffer, D. (2019). Finding Common Ground: A Method for Measuring Recent Temporal Context in Analyses of Complex, Collaborative Thinking. In K. Lund, G. Niccolai, E. Lavoué, C. Hmelo-Silver, G. Gweon, \& M. Baker (Eds.), A Wide Lens: Combining Embodied, Enactive, Extended, and Embedded Learning in Collaborative Settings (Vol. 1, pp. 136-143). International Society of the Learning Sciences.

Shaffer, D. (2017). Quantitative Ethnography. Cathcart Press.

Shaffer, D. W., Collier, W., \& Ruis, A. (2016). A Tutorial on Epistemic Network Analysis: Analyzing the Structure of Connections in Cognitive, Social, and Interaction Data. Journal of Learning Analytics, 3, 9-45.

Siahpush, M. (1999). Postmodern attitudes about health: A population-based exploratory study. Complementary Therapies in Medicine, 7(3), 164-169.

Stratton, T., \& McGivern-Snofsky, J. (2008). Toward a sociological understanding of complementary and alternative medicine use. J Altern Complement Med., 14(6), 777-783.

Sullivan, S., Warner-Hillard, C., Eagan, B., Thompson, R., Ruis, A. R., Haines, K., Pugh, C., Williamson Shaffer, D., \& Jung, H. S. (2018). Using Epistemic Network Analysis to Identify Targets for Educational Interventions in Trauma Team Communication. Surgery, 163, 938-943.

Swiecki, Z., Lian, Z., Ruis, A. R., \& Shaffer, D. W. (2019). Does Order Matter? Investigating Sequential and Cotemporal Models of Collaboration. In K. Lund, G. Niccolai, E. Lavoué, C. Hmelo-Silver, G. Gweon, \& M. Baker (Eds.), A Wide Lens: Combining Embodied, Enactive, Extended, and Embedded Learning in Collaborative Settings (Vol. 1, pp. 112-119). International Society of the Learning Sciences.

Tascilar, M., Jong, F. A. de, Verweij, J., \& Mathijssen, R. H. J. (2006). Complementary and Alternative Medicine During Cancer Treatment: Beyond Innocence. The Oncologist, 11(7), 732-741.

Thompson, C. (2003). Natural health discourses and the therapeutic production of consumer resistance. The Sociological Quarterly, 44(1), 81-107.

Thomson, P., Jones, J., Browne, M., \& Leslie, S. (2014). Psychosocial factors that predict why people use complementary and alternative medicine and continue with its use: A population based study. Complementary Therapies in Clinical Practice, 20(4), 302-310.

Thorne, S., Paterson, B., Russell, C., \& Schultz, A. (2002). Complementary/alternative medicine in chronic illness as informed self-care decision making. Int J Nurs Stud., 39, 671-683. 
Vincent, C., \& Furnham, A. (1996). Why do patients turn to complementary medicine? An empirical study. Br J Clin Psychol., 35(1), $37-48$.

White, M., \& Verhoef, M. (2006). Cancer as part of the journey: The role of spirituality in the decision to decline conventional prostate cancer treatment and to use complementary and alternative medicine. Integrative Cancer Therapies, 5(2), 117122.

Wieland, L., Manheimer, E., \& Berman, B. (2011). Development and classification of an operational definition of complementary and alternative medicine for the Cochrane collaboration. Altern Ther Health Med, 17(2), 50-59.

Wooldridge, A., \& Haefli, R. (2019). Using Epistemic Network Analysis to Explore Outcomes of Care Transitions. In B. Eagan, M. Misfeldt, \& A. Siebert-Evenstone (Eds.), Advances in Quantitative Ethnography: First International Conference, ICQE 2019, Madison, WI, USA, October 20-22, 2019, Proceedings (pp. 245-256). Springer.

Zörgő, S., \& Olivas Hernández, O. (2018). Patient Journeys of Nonintegration in Hungary: A Qualitative Study of Possible Reasons for Considering Medical Modalities as Mutually Exclusive. Integrative Cancer Therapies, 17(4), 1270-1284.

Zörgő, S., Purebl, G., \& Zana, Á. (2018). A Qualitative Study of Culturally Embedded Factors in Complementary and Alternative Medicine Use. BMC Complementary and Alternative Medicine.

Zörgö, S., Swiecki, Z., \& Ruis, A. R. (2021). Exploring the Effects of Segmentation on Semi-Structured Interview Data with Epistemic Network Analysis. In Advances in Quantitative Ethnography. Communications in Computer and Information Science Series: Vol. 1312. (Eds. Ruis AR and Lee, SB., pp. 78-90). Springer Nature. 